\title{
DISCUSSION
}

\section{Lateral earth pressures in constrained landslides}

\author{
BALZ FRIEDLI*, DOMINIK HAUSWIRTH†, ALEXANDER M. PUZRIN $\dagger$, SHIGUO XIAO \\ and TINGJUN CHEN§
}

\section{Contribution by Shiguo Xiao and Tingjun Chen}

The classically kinematical limit analysis method was utilised by the authors to analyse lateral earth pressures in constrained landslides (Friedli et al., 2017). A closed-form solution of earth pressure on retaining structures used to reinforce a planar landslide with a weak slip surface parallel to the slope was given. However, the kinematically admissible translational mechanism proposed by the authors attracted the discussers' attention.

The authors assumed a kinematically admissible translational mechanism involved in three rigid blocks in the paper (Fig. 4(a)). But in fact, the mechanism depends considerably on flexible rigidity of the retaining structures. In order to illustrate the problem, taking case 2 (Fig. 12(b)) as an example, the discussers computed failure mechanisms of upslope soil of the retaining structure with various thicknesses by using the Flac3D program (ICG, 2012). The soil and retaining structure properties of the numerical model (see Fig. 16) are shown in Table 3. The inclination of the slope surface is $20^{\circ}$ and the height of the retaining structure is $20 \mathrm{~m}$. A weak layer with $7^{\circ}$ dip angle was simulated as the frictional interface between the sliding and stable layers. The soil-structure interface was assumed as a completely rough surface. The slope model was divided into eight-node hexahedron elements, which produced 10370 elements in all. The elastic-perfectly plastic constitutive model and Mohr-Coulomb yield criterion were used for the slope material. The associated flow rule was adopted as well, to agree with the basic principle of the upper-bound limit analysis. The retaining structure was taken as an elastic body

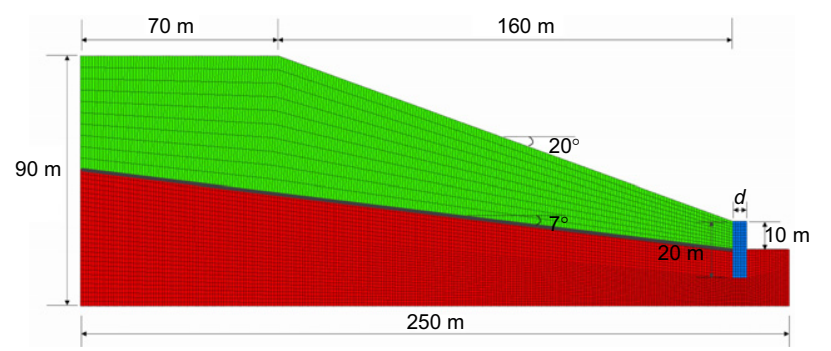

Fig. 16. Cross-section of numerical simulation model of the example with $1 \mathrm{~m}$ thickness out of plane

\footnotetext{
* Institute for Geotechnical Engineering, ETH Zurich, Switzerland (Orcid:0000-0003-2178-2761).

$\dagger$ Institute for Geotechnical Engineering, ETH Zurich, Switzerland. \$ Key Laboratory of Transportation Tunnel Engineering and Key Laboratory of High-speed Railway Engineering, Ministry of Education, Southwest Jiaotong University, Chengdu, P. R. China. $\S$ Department of Geological Engineering, Southwest Jiaotong University, Chengdu, P. R. China.
}

with thickness $d$ varying from $1 \mathrm{~m}$ to $5 \mathrm{~m}$. As shown in Fig. 17, the failure mechanisms of the retained slope obtained using Flac3D by way of the shear strength reduction method are various with the increase of the structure thickness. If the thickness is $1-3 \mathrm{~m}$, the corresponding failure mechanisms cannot be regarded as three simple rigid blocks bounded by planar discontinuity, but as close to two blocks with a curved discontinuity bound. Hence, under the condition that the flexible rigidity of the retaining structure is high (e.g. the thickness is $5 \mathrm{~m}$ in the example), the failure mechanism can be assumed to be that proposed by the authors. However, if the flexible rigidity is relatively low (e.g. the thickness is $1 \mathrm{~m}$ in the example), the mechanism proposed by the authors is not reasonable. That is to say, there are significant differences of the kinematically admissible failure mechanism of the retained soil between the flexible and rigid retaining structures.

\section{Authors' reply}

The authors would like to thank the discussers for their interest in this work and their contribution.

The discussers used a numerical tool for investigating the analytically formulated problem of lateral pressure in a constrained landslide. They varied the wall thickness in a strength reduction analysis between $1 \mathrm{~m}$ and $5 \mathrm{~m}$ in order to study a potential influence of flexural rigidity on the limiting landslide pressure. While they observed the authors' proposed landslide failure mechanism for walls with large thickness, for walls with small thickness their calculations showed a different failure pattern. The discussers concluded that the failure mechanism in a constrained landslide depends on the flexural rigidity and that the mechanism deviates from the authors' proposed landslide failure mechanism for walls with low flexural rigidity or thickness.

In the authors' opinion the varying failure mechanism observed by the discussers is a result of insufficient resistance against rotational failure of the walls having a small wall thickness. This reply to the discussion is structured in two parts. In the first part the basic assumptions of the authors' work are restated briefly. In the second part, the observations made by the discussers are analysed and an explanation is given for the conditions under which they materialise.

\section{Basic assumptions}

The authors' work investigated the limiting lateral earth pressure in constrained landslides acting either within the sliding layer or on the constraining obstacle - for example, a retaining wall. This limiting pressure is described as an ultimate limit state by the failure of the soil above the constraining obstacle and can only be reached if the obstacle is able to provide sufficient bearing resistance. Measures and methods on how to transfer the landslide pressure through the obstacle into the stable ground were not within the scope 
Table 3. Soil and retaining structure properties of the numerical simulation model

\begin{tabular}{l|c|c|c|c|c}
\hline & Unit weight: $\mathrm{kN} / \mathrm{m}^{3}$ & Cohesion: kPa & Internal friction angle: degrees & Elastic modulus: MPa & Poisson's ratio \\
\hline Sliding layer & 20 & 0 & 30 & 30 \\
Stable layer & 22 & 30 & 45 & $0 \cdot 4$ \\
Weak layer & $/$ & 0 & 7 & $0 \cdot 3$ \\
Retaining structure & 25 & $/$ & $/$ & $/$ & 300 \\
\hline
\end{tabular}

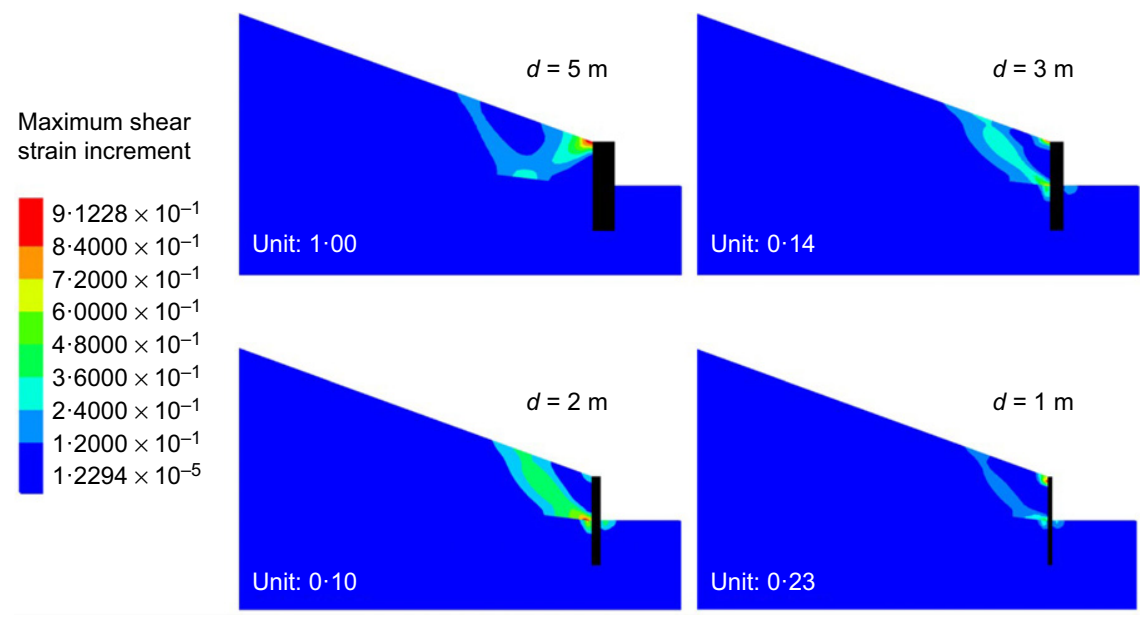

Fig. 17. Contours of maximum shear strain increment of the example under various thicknesses of the retaining structure obtained using Flac3D program

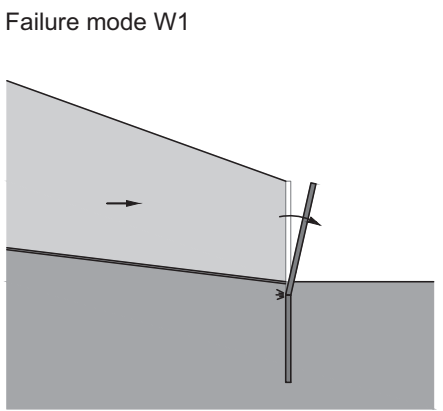

(a)

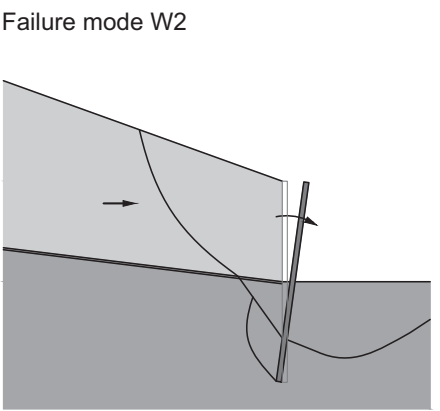

(b)

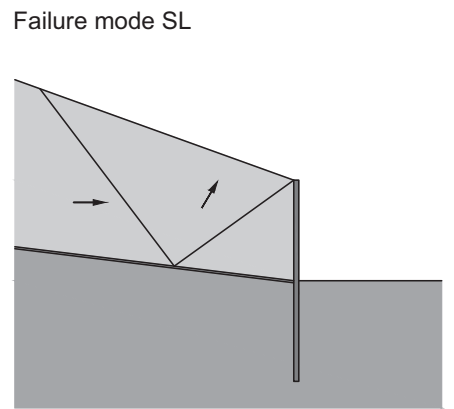

(c)

Fig. 18. Possible failure modes: (a) internal failure of the wall (W1); (b) external failure of the wall (W2); (c) failure of the sliding layer (landslide pressure SL)

of the study. The problem of lateral pressures was solved using the upper and lower bound theorem of limit analysis and its underlying assumptions - for example, an elasticperfectly plastic material, convex yield surface, associated flow and small deformations at failure. Within this framework, the flexural rigidity has no influence on the landslide pressure, provided the wall deformations are sufficiently small to regard the deformed geometry as unaltered and the wall is able to bear the landslide pressure. Note that for the particular case analysed by the discussers, with non-parallel ground and a weak layer surface, the authors' work only provides an upper bound solution.

A stabilising retaining wall in a landslide, as investigated by the discussers, may fail in principle owing to the loss of internal (e.g. bending failure, mode W1) or external equilibrium (e.g. loss of rotational stability, mode W2), as shown in Figs 18(a) and 18(b), respectively. In the latter case, the limit state occurs mainly in the stable layer below the retaining structure and only to a small extent within the sliding layer. In contrast, the authors' work addressed the limiting pressure within the sliding layer or the pressure acting on the constraining obstacle, assuming that the constraining obstacle does not fail under the loads of the landslide pressure - that is, excluding the failure modes $\mathrm{W} 1$ and W2. In this case, failure has to take place entirely within the sliding layer, as illustrated with failure mode SL in Fig. 18(c). Hence, the solution provided can be regarded as a maximum load for which a retaining structure has to be designed. If the wall fails before the landslide pressure is reached, the failure mechanism within the sliding layer can be different and the corresponding lateral pressure on the wall will be smaller than the landslide pressure. Below, the authors show that this was presumably the case in the discussers' analysis for thinner walls.

\section{Analysis}

Based on their numerical study the discussers observed different mechanisms depending on the wall thickness. This observation led them to the conclusion that the flexural 


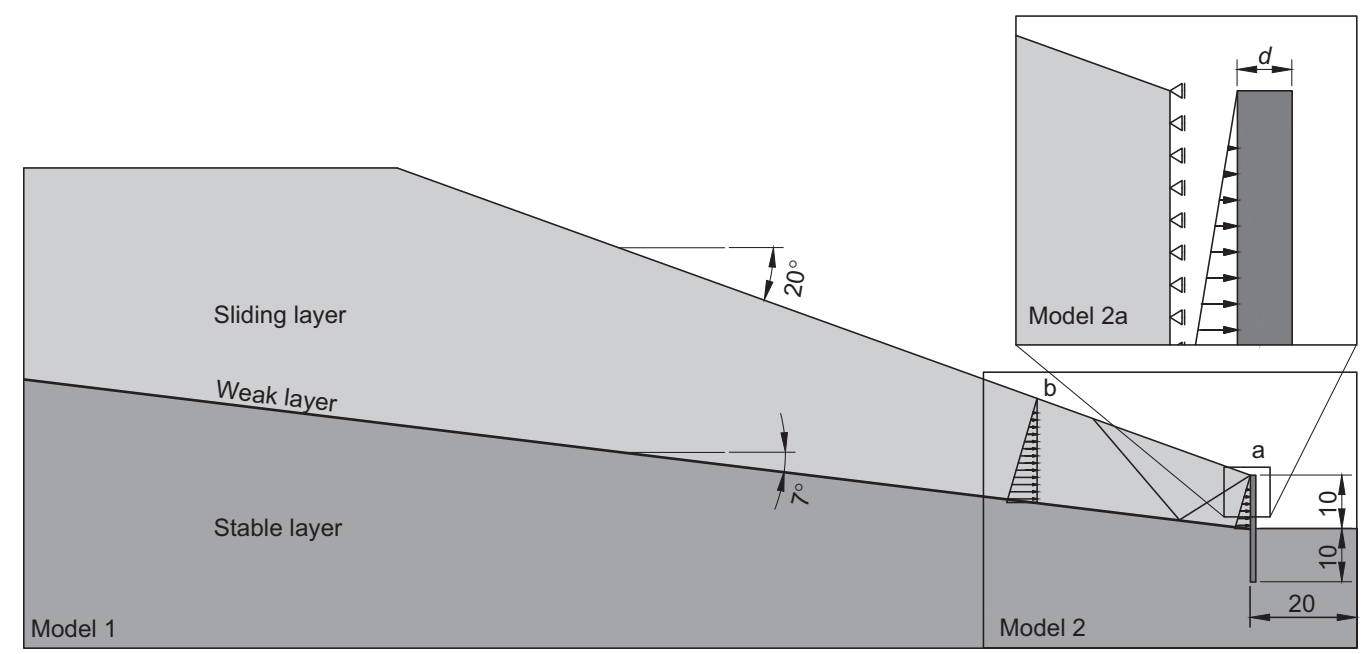

Fig. 19. Geometry of the analysed models

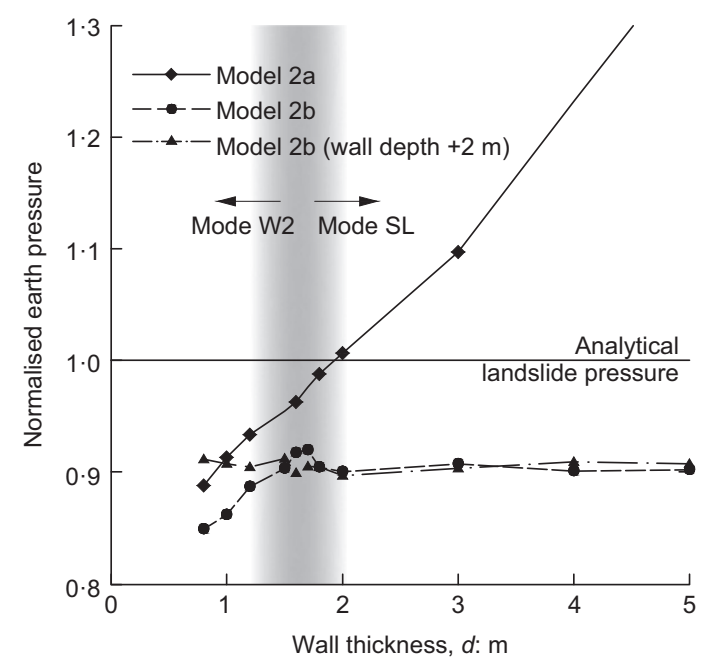

Fig. 20. Results of the numerical analysis

rigidity of the wall is controlling the type of failure mechanism. However, by varying the wall thickness, not only flexural rigidity but also the resistance against rotational failure of the rough wall is modified. Below a certain wall thickness, the unsupported wall may not be able to bear the landslide pressure any longer and, consequently, the failure mode may change from SL to W2. In order to validate this hypothesis, the authors utilised two numerical models (Fig. 19): model 1 is identical to the discussers' model with the same slope and weak layer inclinations, wall dimensions, material and interface parameters; model 2 is a smaller part of model 1. In contrast to the discussers' model, which used a strength reduction analysis, the authors applied in model 2 a triangularly distributed load to simulate approximately the landslide pressure. In model 2a the load was applied directly to the retaining wall, with the sliding layer separated from the wall using a zero horizontal displacement boundary condition. This model was used to find the resistance of the wall against rotational failure according to failure mode W2. In model $2 b$, the wall and the sliding layer were connected and the load was applied at the distance of $40 \mathrm{~m}$ above the wall. This model was used to investigate failure mechanisms in the sliding layer above the wall. Note that the results presented are based on a rather crude numerical analysis, which is used for a phenomenological explanation of the

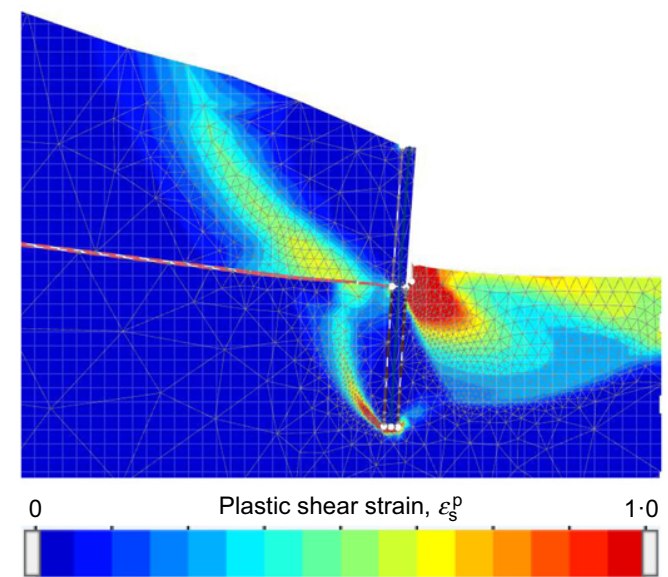

(a)

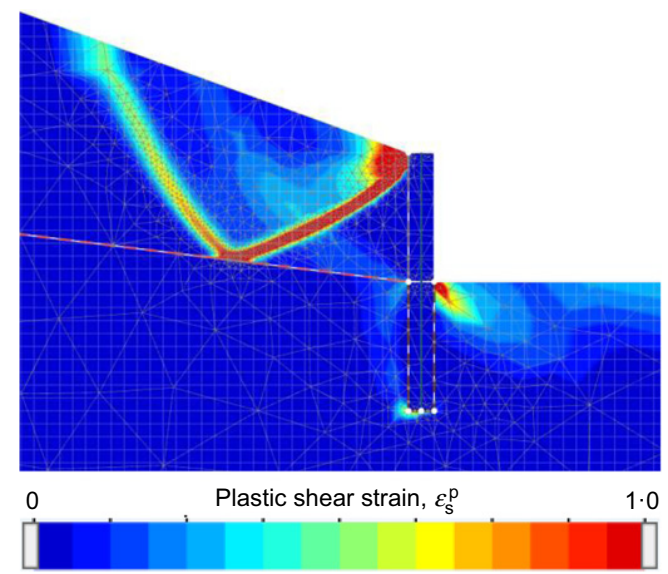

(b)

Fig. 21. Plastic shear strains of model $2 \mathrm{~b}$ with: (a) $1 \mathrm{~m}$ wall thickness, failure according to mode W2; (b) $2 \mathrm{~m}$ wall thickness, failure according to mode SL

discussers' observations. Absolute values may have limited accuracy.

Figure 20 shows the failure load resulting from model $2 \mathrm{a}$, normalised with the analytical upper bound solution proposed in the original paper, for varying wall thickness, $d$. It is shown that the resistance of the wall indeed increases with the wall thickness. Furthermore, it is shown that for a 
wall thickness below 1-2 $\mathrm{m}$, the failure load of the wall is lower than the load according to the analytical landslide pressure solution. The authors conclude that, in the special case analysed by the discussers, the mechanism proposed in the original paper, with failure in the sliding layer, cannot be reached for smaller wall thicknesses owing to the preceding rotational failure of the wall. This finding is supported by the results from model $2 \mathrm{~b}$ in Fig. 20, where for the wall with a thickness larger than $1-2 \mathrm{~m}$ a failure load close to the analytical upper bound solution was found, which is independent of the wall thickness. The corresponding plastic shear strains indicate a failure mechanism, which closely resembles the proposed mechanism in the paper under discussion (failure mode SL, Fig. 21(b)). For thinner walls, however, the failure load drops with decreasing wall thickness and is close to the one calculated using model $2 \mathrm{a}$. In these cases, the plastic shear strains show a mechanism corresponding to a rotational failure of the wall (failure mode W2, Fig. 21(a)). The same change of the failure mode was also observed when a strength reduction analysis was performed using model 1 , similar to the one conducted by the discussers.

It is important to note that the transition from failure mode W2 to mode SL is not caused by the change of flexural rigidity, as implied by the discussers, but is caused by the increase of the resistance against rotational failure of the retaining wall. This was confirmed by changing the Young's modulus of the retaining wall, while keeping its thickness constant. In contrast, the variation of the wall thickness not only changes the flexural rigidity, but has also an effect on the resistance of the wall against rotational failure. Furthermore, an elongation of the embedded wall depth by $2 \mathrm{~m}$, which increases the resistance against rotational failure, always leads to the failure mode SL regardless of the wall thicknesses (Fig. 20, dashed line with triangles). The same observation was made when the rotational wall failure was excluded by increasing the strength in the stable layer or treating it as elastic or rigid.
Summary

In addition to the explanation for the discussers' observation provided above, the authors would like to summarise the following key points

(a) The paper under discussion treats the limiting pressure in constrained landslides, presuming a sufficient resistance of the constraining obstacle to bear this pressure.

(b) A retaining structure with resistance lower than the landslide pressure does not act as an obstacle in a long-term consideration. Therefore, different mechanisms (e.g. mode W1 or W2) can be observed. In these cases, failure takes place mainly outside the sliding layer. Such failure modes were, however, not the subject of the original paper.

(c) The flexural rigidity of the wall is not controlling the mode of failure (within small deformations) - it is the internal and external resistance of the wall with respect to the landslide pressure.

Given that the constraining obstacle does not fail, it can be concluded that the landslide pressure will be reached with a failure mechanism resembling the one proposed in the paper under discussion.

\section{ACKNOWLEDGEMENT}

The numerical results presented in this contribution were obtained by applying elastic-plastic failure load analysis using the finite-element code Optum 2G (Optum Computational Engineering, Copenhagen, Denmark).

\section{REFERENCES}

Friedli, B., Hauswirth, D. \& Puzrin, A. M. (2017). Lateral earth pressures in constrained landslides. Géotechnique 67, No. 10, 890-905, https://doi.org/10.1680/jgeot.16.P.158.

ICG (Itasca Consulting Group) (2012). FLAC3D 5.0 manual. Minneapolis, MN, USA: Itasca Consulting Group. 